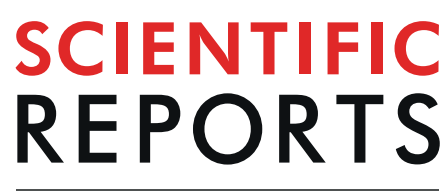

natureresearch

\title{
OPEN Author Correction: Human brain patterns underlying vigilant attention: impact of sleep debt, circadian phase and attentional engagement
}

\author{
Micheline Maire ${ }^{1,2,3}$, Carolin F. Reichert ${ }^{1,3}$, Virginie Gabel ${ }^{1,3}$, Antoine U. Viola ${ }^{1,3,8}$, \\ Christophe Phillips $\mathbb{1}^{4}$, Christian Berthomier ${ }^{7}$, Stefan Borgwardt ${ }^{5,6}$, Christian Cajochen ${ }^{1,3}$ \& \\ Christina Schmidt ${ }^{1,4}$
}

Correction to: Scientific Reports https://doi.org/10.1038/s41598-017-17022-9, published online 17 January 2018

The original version of this Article contained errors in the spelling of the authors Micheline Maire, Carolin F. Reichert, Virginie Gabel, Antoine U. Viola, Christophe Phillips, Christian Berthomier, Stefan Borgwardt, Christian Cajochen \& Christina Schmidt which were incorrectly given as M. Maire, C. F. Reichert, V. Gabel, A. U. Viola, C. Phillips, C. Berthomier, S. Borgwardt, C. Cajochen \& C. Schmidt respectively.

These errors have now been corrected in the PDF and HTML versions of the Article.

(i) Open Access This article is licensed under a Creative Commons Attribution 4.0 International License, which permits use, sharing, adaptation, distribution and reproduction in any medium or format, as long as you give appropriate credit to the original author(s) and the source, provide a link to the Creative Commons license, and indicate if changes were made. The images or other third party material in this article are included in the article's Creative Commons license, unless indicated otherwise in a credit line to the material. If material is not included in the article's Creative Commons license and your intended use is not permitted by statutory regulation or exceeds the permitted use, you will need to obtain permission directly from the copyright holder. To view a copy of this license, visit http://creativecommons.org/licenses/by/4.0/.

(c) The Author(s) 2019

\footnotetext{
${ }^{1}$ Centre for Chronobiology, Psychiatric Hospital of the University of Basel, Basel, Switzerland. ${ }^{2}$ Institute of Primary Health Care (BIHAM), University of Bern, Bern, Switzerland. ${ }^{3}$ Transfaculty Research Platform Molecular and Cognitive Neurosciences, University of Basel, Basel, Switzerland. ${ }^{4}$ GIGA-CRC In Vivo Imaging, University of Liège, Liège, Belgium. ${ }^{5}$ Medical Image Analysis Center, University Hospital of Basel, Basel, Switzerland. ${ }^{6}$ Department of Psychiatry, University Hospital of Basel, Basel, Switzerland. ${ }^{7}$ PHYSIP SA, Paris, France. ${ }^{8}$ PPRS, Paris, France. Christian Cajochen and Christina Schmidt contributed equally. Correspondence and requests for materials should be addressed to C.C. (email: christian.cajochen@upkbs.ch)
} 